\title{
Electrical and optical properties of Makrofol DE 1-1 polymeric films induced by gamma irradiation
}

\author{
Y. S. Rammah", S. E. Ibrahim and E. M. Awad
}

\begin{abstract}
Background: A set of Makrofol DE 1-1 polymeric samples was irradiated to different gamma doses. The dielectric response of irradiated polymers was investigated at a wide range of frequencies at room temperature. The modification induced in optical properties include the optical band gap ( $\left.E_{\text {optical }}\right)$, number of carbon per cluster $(M)$, number of carbon per length $(N)$, and refractive index $(n)$ for irradiated samples were studied as well.

Methods: The dielectric constant $(\dot{\varepsilon})$, dielectric loss factor $\left(\varepsilon^{\prime}\right)$, loss tangent (tan $\left.\delta\right)$, the ac electrical conductivity ( $\left.\sigma_{a c}\right)$, and impedance (Z) were measured in the frequency range from (100) $\mathrm{Hz}$ to $(100) \mathrm{MHz}$. The optical band gap energy and its corresponding parameters for the samples were evaluated using ineffective thickness method (ITM).

Results: Gamma-ray irradiation had a considerable effect on the optical band gap energy for Makrofol DE 1-1 polymer detector film, which decreases from 4.20 to $3.96 \mathrm{eV}$ and 4.35 to $4.16 \mathrm{eV}$ for indirect and direct allowed transitions in ITM method, respectively, while it decreases from 4.40 to $4.20 \mathrm{eV}$ in DITM method as a result of $\gamma$-ray dose increases. ( $n$ ) of all studied samples is considerably high. $(N)$ and $(M)$ for the direct transition are lower than that for the indirect transition and their values increase with increasing gamma dose.

Conclusion: The results reveal that Makrofol DE1-1 polymer can be used as gamma dosimeter. Furthermore, Makrofol DE1-1 has much greater resistance to radiation damage; the attained results suggested strongly the applicability of Makrofol DE1-1 polymer to be used in medical products applications and suitable for optoelectronic and photoelectric devices.
\end{abstract}

Keywords: Makrofol-DE1-1, ү-dose, ac electrical conductivity, Dielectric constant, Dielectric loss, Optical energy gap

\section{Highlights}

- Makrofol DE1-1 polymeric detectors have been irradiated with $\gamma$-irradiation with different doses.

- Electrical parameters of the pristine and irradiated samples were calculated.

- UV-visible spectra have been studied and the optical energy band gap as well as refractive index of un-irradiated and irradiated samples have been calculated.

- Number of carbon atoms per conjugate length and cluster have been calculated.

\footnotetext{
* Correspondence: dr_yasser1974@yahoo.com

Physics Department, Faculty of Science, Menoufia University, Shebin El-Koom, Menoufia 32511, Egypt
}

\section{Background}

Polymeric materials were and still have an interesting point of study, because its structure could be significantly changed by radiation. Thus, polymer composites and structure have steadily gained growing importance in the last few decades. The structure of such materials has been accomplished through electrical conductivity measurements (Zahran et al. 1981; Kumar et al. 2006). Irradiated polymeric materials with ionizing radiation such as $\gamma$-rays, $\mathrm{X}$-rays, accelerated electrons, and ion beams leads to the formation of very reactive intermediates products like excited states, ions, and free radicals, which result in rearrangements and/or formation of new bonds. The effects of these reactions lead to the formation of oxidized products, grafts, scission of main chain (degradation), or cross-linking beside its effects on the 
optical, thermal, and electrical behavior of such polymer (Zahran et al. 1981; Saad et al. 2014; Fares 2011; Tayel et al. 2015; Rammah 2015, 2017; Al-Amri et al. 2017; Alfaramawi et al. 2017; Alsalhi et al. 2017; Jaleh et al. 2017; Rammah and Abdalla 2017; Rammah and Awad 2018; Saad et al. 2018; Abdul-Kader et al. 2018). Degradation and cross-linking occur simultaneously, and the outcome of the process is determined by a competition between the reactions (Kumar et al. 2006; Sinha et al. 2004). Oxidation and degradation occur gradually with increasing irradiation dose. Polycarbonate (PC) is a well-known engineering thermoplastic with an excellent balance of optical, physical, mechanical, and processing characteristics (Chung 1997; Cheng 2003). Polycarbonate detectors are used as a particle track detector for neutrons and alpha particles (Nouh et al. 2004; Price 2005; Awad et al. 2007; Awad et al. 2008; Rammah et al. 2018). Moreover, radiation effects on dielectric properties are of particular interest to science and technology and they have many applications in modern engineering (Basha et al. 1996; Ahmed et al. 2001; Kecskemeti et al. 2006; Hanafy 2008), and the effect of ion irradiation on the dielectric properties of polymers has been also studied earlier (Yoshida et al. 1980; Chailan et al. 1997; Martinez-Pardoma et al. 1998; Phukan et al. 1999; Fares 2011).

Therefore, the goal of the present work is to study the effect of gamma irradiation on electrical and optical properties of Makrofol DE 1-1 polymer detector. The dielectric constant $\left(\varepsilon^{\prime}\right)$, dielectric loss $\left(\varepsilon^{\prime \prime}\right)$, ac conductivity $\left(\sigma_{\mathrm{ac}}\right)$ were measured and discussed. The optical energy band ( $\left.E_{\text {optical }}\right)$, number of carbon atoms per cluster $(M)$, number of carbon atoms per length $(N)$, and refractive index $(n)$ were calculated using ineffective thickness method (ITM) and derivation of ineffective thickness method (DITM) methods.

\section{Methods and theoretical considerations Dielectric properties}

The parameters of dielectric studies like the dielectric constant, $\varepsilon^{\prime}$, dielectric loss, $\varepsilon^{\prime \prime}$, and ac conductivity, $\sigma_{\mathrm{ac}}$ reveals the electro-optic coefficient of the material (Ignatius et al. 2014). These parameters have been calculated by means of the following relations:

$$
\begin{aligned}
& \varepsilon^{\prime}=\frac{C d}{\varepsilon_{o} A} \\
& \varepsilon^{\prime \prime}=\varepsilon^{\prime} \tan \delta \\
& \sigma_{a c}=\frac{d}{Z A}
\end{aligned}
$$

where

$C, d, A$, and $\varepsilon_{\mathrm{o}}$ are the capacitance, the thickness, the cross-sectional area of the sample, and the absolute permittivity of vacuum $\left(8.854 \times 10^{-9} \mathrm{~F} / \mathrm{cm}\right)$, respectively. $\tan \delta$ is the dissipation factor and $Z$ is the impedance of the sample.

\section{Optical properties}

The optical properties for amorphous and crystalline materials like the optical energy band gap, $E_{\text {optical }}$ and refractive index, $n$ were studied by investigating the samples absorption spectra in the UV region. The electrons in the valence bands interact with electromagnetic waves and are raised to the conduction band. The optical transitions occur by two different ways; one is the indirect (forbidden or allowed) transition, whereas the other is the direct (forbidden or allowed) transition. In this study, the optical band gap energy for the samples were evaluated using ineffective thickness method (ITM). In this method, the optical band gap can be determined using data of the film absorbance spectra and independent on the samples thickness (Ghobadi 2016). Tauc's formula (Tauc 1974) which modified by Mott and Davis (Mott and Davies 1979) as follows:

$$
[\alpha(v) E]^{1 / m}=G\left(E-E_{\text {optical }}\right)
$$

where $(G)$ is a constant, $(E=h v)$ is the incident photons energy, and ( $\left.E_{\text {optical }}\right)$ is optical energy band. $(m)$ is the power characterizes electronic transition, and equal 0.5 and 2 for direct and indirect allowed transition, respectively. $\alpha(v)$ is optical absorption coefficient defined by Beer-Lambert's law as follows:

$$
\alpha(v)=\frac{2.303 A(\lambda)}{t}
$$

where $A(\lambda)$ and $t$ are the film absorbance and thickness, respectively. Substitute Eq. (5) into Eq. (4), one can get:

$$
\left(\frac{2.303 A(\lambda)}{t} E\right)^{1 / m}=G\left(E-E_{\text {optical }}\right)
$$

Eq. (6) can be rearranged as follows:

$$
\left(\frac{2.303}{t}\right)^{1 / m}(A(\lambda) E)^{1 / m}=G\left(E-E_{\text {optical }}\right)
$$

Let the constant $\left(\frac{2.303}{t}\right)^{1 / m}$ equals $K$, then Eq. (7) writes as follows:

$$
(A(\lambda) E)^{1 / m}=\frac{G}{K}\left(E-E_{\text {optical }}\right)
$$

Substitute the ratio $\frac{G}{K}$ by new constant $C$, then

$$
(A(\lambda) E)^{1 / m}=C\left(E-E_{\text {optical }}\right)
$$

Equation (9) shows that optical band gap ( $\left.E_{\text {optical }}\right)$ determination depends only on absorbance $(A(\lambda))$ spectra 
instead of the absorption coefficient and avoiding any needs to film thickness. Then the values of optical energy band gap for the studied samples can be determined directly from extrapolating the linear region of $(A(\lambda) \mathrm{E})^{1 / \mathrm{m}}-E$ curve at $(A(\lambda) \mathrm{E})^{1 / \mathrm{m}}=0$.

The exact optical energy band gap of the studied samples can be evaluated by another method called derivation of ineffective thickness method (DITM), which is proposed to estimate of the exact optical band gap (Ghobadi 2016). In this method, the measure absorbance spectrum of the sample is needed only as well. Starting from ineffective thickness method (ITM), Eq. (9) can be rewritten as follows:

$$
(A(\lambda) E)=C\left(E-E_{\text {optical }}\right)^{m}
$$

Taking $\ln$ of both sides of Eq. (10)

$$
\ln [A(\lambda) E]=\ln \left[C\left(E-E_{\text {optical }}\right)^{m}\right]
$$

Then

$$
\ln [A(\lambda) E]=m \ln (C)+m \ln \left(E-E_{\text {optical }}\right)
$$

Taking derivation of Eq. (12) with respect to $E$ gives:

$$
\frac{d\{\ln [A(\lambda) E]\}}{d E}=\frac{m}{E-E_{\text {optical }}}
$$

Plotting $\frac{d\{\ln [A(\lambda) E]\}}{d E}$ versus $E$, a discontinuity at $(E=$ $\left.E_{\text {optical }}\right)$ will be found and the optical band gap can be estimated directly at this point.

Once the optical energy band gap of the studied samples were determined, their refractive index can be estimated (Dimitrov and Sakka 1996; Ali et al. 2017):

$$
\left(\frac{n^{2}-1}{n^{2}+2}\right)=1-\left(\frac{E_{\text {optical }}}{20}\right)^{1 / 2}
$$

For a linear structure, the number of carbon atoms per conjugation length, $(N)$ is given by (Fink and Hnatowicz 2007):

$$
N=\frac{2 \beta \pi}{E_{\text {optical }}}
$$

Where the value of $\beta$ is taken to be $-2.9 \mathrm{eV}$ as it is associated with $\pi-\pi^{*}$ optical transitions in $-\mathrm{C}=\mathrm{C}$ - structure and $2 \beta$ gives the band structure energy of a pair of adjacent $\pi$ sites. Finally, the number of carbon atoms per cluster $(M)$ is given by (Fink and Hnatowicz 2007):

$$
M=\left(\frac{34.3}{E_{\text {optical }}}\right)^{2}
$$

\section{Experimental procedures}

Makrofol DE 1-1 (bisphenol-A polycarbonate) nuclear track detectors (NTDs) of chemical composition $\left[\begin{array}{lll}\mathrm{C}_{16} & \mathrm{H}_{14} & \mathrm{O}_{3}\end{array}\right]$ with thickness of $175 \mu \mathrm{m}$ and density $1.2 \mathrm{~g} / \mathrm{m}^{3}$ were used. Chemical structure (repeating unit) of Makrofol DE 1-1 polycarbonate detector is shown in Fig. 1. The samples were supplied by Bayer AG Leverkusen, Germany. The polymeric detector samples were cut to a size of $2 \times 2 \mathrm{~cm}^{2}$ and irradiated with different $\gamma$-ray doses, viz. 1, 10, and $100 \mathrm{kGy}$ using $1.25 \mathrm{MeV}^{60} \mathrm{Co}$ gamma cell irradiation facility of type MC-20 with dose rate $4 \mathrm{kGy} / \mathrm{h}$ fabricated by the Russian Atomic Energy at Egyptian Atomic Energy Authority (EAEA). The detector samples were positioned at the center of the driving belt and the irradiation process was achieved automatically. The samples were irradiated for different times to maintain the desired doses.

Pristine and $\gamma$-irradiated samples were subjected to optical measurements using UV-visible spectrometer (UVS-2700) in the wavelength range from 190 to $1100 \mathrm{~nm}$.

The electrical measurements of all samples were carried out at room temperature using an LCR (Hioki 3520 HiTester Meter Bridge) over the frequency range of $100 \mathrm{~Hz}-100 \mathrm{kHz}$. The good electrical contact during measurements was made by applying an air-drying type silver paint on the opposite sides of the bulk samples. By using the measured values of capacitance $C$, dielectric loss factor $\tan \delta$, and dimensions of the samples, the dielectric constant, $\varepsilon^{\prime}$, dielectric loss, $\varepsilon^{\prime \prime}$, and ac conductivity, $\sigma_{\mathrm{ac}}$ have been calculated using Eqs. (1)-(3).

\section{Results}

The dielectric constant $\varepsilon^{\prime}$ and dielectric loss $\varepsilon^{\prime \prime}$ of un-irradiated and irradiated samples were found high at low-frequency region (nearly below $20 \mathrm{kHz}$ ), and their values increase with increasing $\gamma$-irradiation dose. The ac conductivity of the studied polymer increases with increasing the frequency $\gamma$-irradiation dose as well. Gamma-ray irradiation had a considerable effect on the optical band gap energy for Makrofol DE 1-1 polymer detector film, which decreases from 4.20 to $3.96 \mathrm{eV}$ and 4.35 to $4.16 \mathrm{eV}$ for indirect and direct allowed transitions in ITM method, respectively, while it decreases from 4.40 to $4.20 \mathrm{eV}$ in DITM method as a result of the increase in $\gamma$-ray dose. The refractive index of all studied samples is considerably high, its values for the direct transition is less than that for the indirect transition and increases with increasing of $\gamma$-dose. The number of carbon atoms per conjugate length, $N$ and the number of

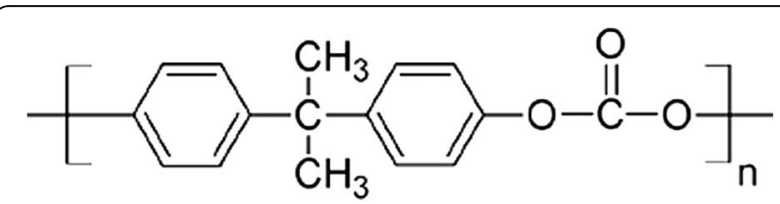

Fig. 1 Chemical structure (repeating unit) of Makrofol DE 1-1 polycarbonate $\left(\mathrm{C}_{16} \mathrm{H}_{14} \mathrm{O}_{3}\right)$ 
carbon atoms per cluster $M$ for the direct band gap was found lower than that for the indirect energy band gap, and their values increase with increasing gamma dose.

\section{Discussion}

\section{Electrical properties}

Figures 2 and 3 depict the dielectric constant $\varepsilon^{\prime}$ and dielectric loss $\varepsilon^{\prime \prime}$ as a function of frequency for Makrofol DE 1-1 polymer irradiated to $\gamma$-ray with doses $0,1,10$, and $100 \mathrm{kGy}$ at room temperature. One can observe that the dielectric constant $\varepsilon^{\prime}$ and dielectric loss $\varepsilon^{\prime \prime}$ of all samples are high in the lower frequency region (nearly below $20 \mathrm{kHz}$ ). However, the region below $1 \mathrm{kHz}$ shows sharper increase. At higher frequencies, the value $\varepsilon^{\prime}$ and $\varepsilon^{\prime \prime}$ were slowly decreased and nearly constant at frequency of $10 \mathrm{kHz}$. It can be attributed to the fact that as the frequency increases, the dipolar molecules in the polymer cannot be able to response further to the increase in the rates of the applied field. The observed increase in dielectric constant $\varepsilon^{\prime}$ and dielectric loss $\varepsilon^{\prime \prime}$ toward the low-frequency region is attributed to the interfacial polarization (Rajendran et al. 2004). Both pristine polymer and irradiated samples show similar trend. Results of Figs. 2 and 3 reveal that an increase in $\varepsilon^{\prime}$ and $\varepsilon^{\prime \prime}$ values with $\gamma$-ray doses. This can be attributed to the formation of some defects sites in the band gaps of polymer because of occurrence of chain scission. Generally, these defects may indicate sign of the existence of charge carriers traps in the band gap of the polymer (Velitchkova et al. 2000; El-Sayed et al. 2004). Therefore, the irradiation increases the ability of the polymer to store charge, i.e., the increase of its dielectric constant (Abdul-Kader et al. 2014).
Figure 4 shows the variation of the Makrofol DE 1-1 samples impedance with frequency for different gamma irradiation doses. It can be noticed that the impedance rapidly decreases by increasing the frequency and the impedance increased by increasing the dose.

Figure 5 illustrates the frequency dependence of ac conductivity, $\sigma_{\mathrm{ac}}$, for irradiated Makrofol DE 1-1 polymer at room temperature. From this figure, one can notice that ac conductivity of samples increases with increasing frequency, which is the common characteristic of disordered materials and increases with increasing gamma irradiation doses. Figures 4 and 5 show consistent data that at high frequency the impedance decreases and the ac conductivity increases.

The variation of the ac conductivity of Makrofol DE 1-1 samples with different gamma doses at different frequencies is presented in Fig. 6. It was found that as the dose increases as the $\sigma_{\mathrm{ac}}$ increases up to $10 \mathrm{kGy}$ and the detector ac conductivity is almost saturated from $10 \mathrm{kGy}$ up to $100 \mathrm{kGy}$. Polymer exposure to gamma irradiation will lead to increase the conductivity of the detector as explained in literature (Singh et al. 2005; Czvikovszky and Hargitai 1999). Interaction of gamma photons with the polymer components will produce a flood of $\delta$-rays, which in turn produce huge number of free radicals leading to scission of polymer chains. With increasing the absorbed doses, the energy deposited in polymer material increases and therefore the number of the conjugated double bonds increases (Hussain et al. 2005). Increasing the conjugated double bonds increases the delocalization of charge carriers, and hence their motion in an applied external electric field. This net polarization produces higher ac conductivity at higher frequency (Jonscher 1977).

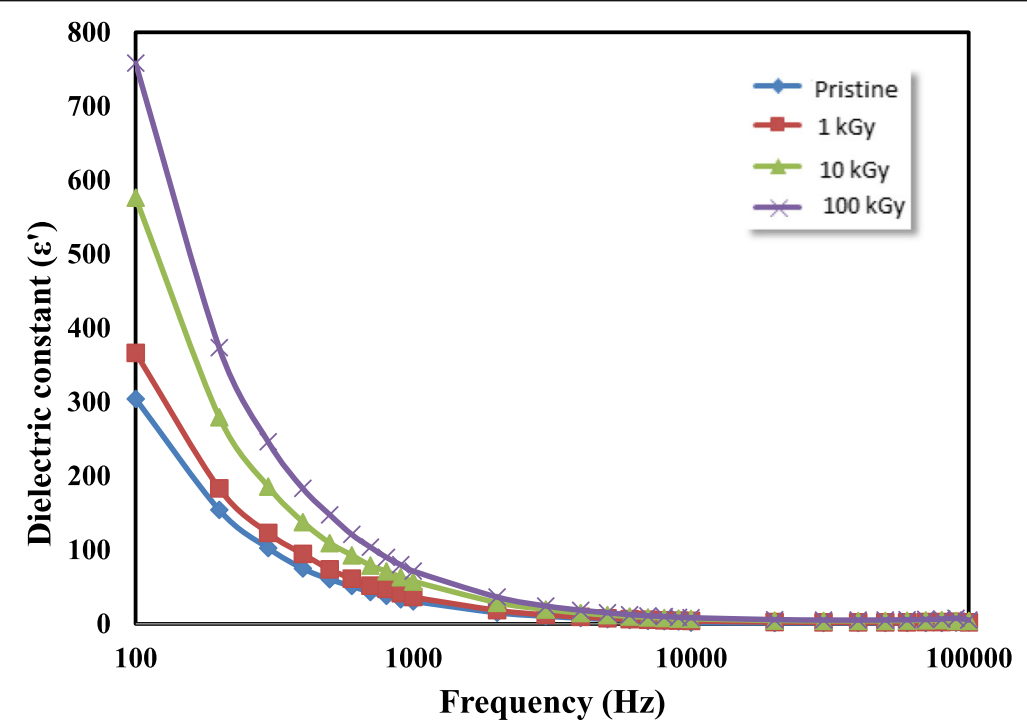

Fig. 2 Frequency dependence of the dielectric constant for the investigated samples at room temperature 


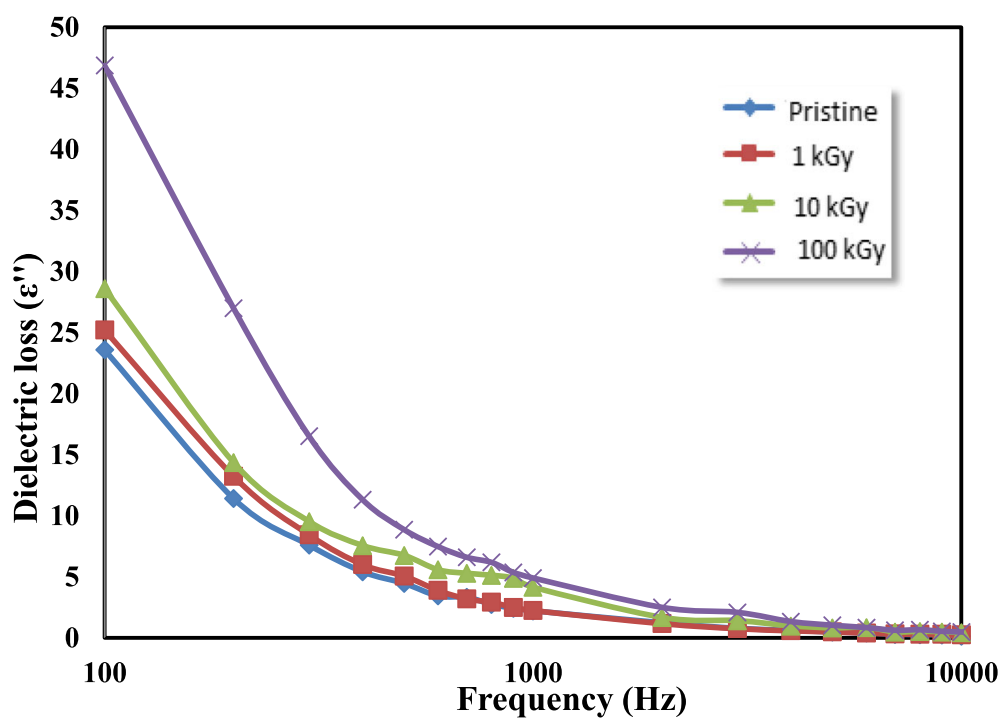

Fig. 3 Frequency dependence of the dielectric loss for the investigated samples at room temperature

\section{Optical properties}

Figure 7 illustrates the experimental UV spectra at 280$400 \mathrm{~nm}$ for pristine and $\gamma$-irradiated Makrofol DE 1-1 samples. Pristine and $1 \mathrm{kGy}$ irradiated samples show the same absorption behavior. Higher $\gamma$-doses (greater than $1 \mathrm{kGy}$ ) lead to formation of color centers appeared as a shoulder superimposed on the UV spectra in the range $310-320 \mathrm{~nm}$. This may be the reason for the color change of plastic from transparent to faint yellowish after $\gamma$-irradiation. The optical energy band gap for the studied samples were determined using ineffective thickness method (ITM) (Ghobadi 2016) by putting the power $m$ in Eq. (9) equals to 0.5 and 2 for direct and indirect allowed transition, respectively. Plotting $(A(\lambda) E)^{2}$ and $(A(\lambda) E)^{1 / 2}$ against
$E$ for direct and indirect allowed transition are shown in Figs. $8 \mathrm{a}-\mathrm{d}$ and $9 \mathrm{a}-\mathrm{d}$, respectively. The values of optical energy band gap for pristine and $\gamma$-irradiated Makrofol DE 1-1 samples were determined directly from extrapolating the linear region of $(A(\lambda) E)^{1 / \mathrm{m}}-E$ curve at $(A(\lambda) E)^{1 / \mathrm{m}}=0$ and the values are tabulated in Table 1.

The optical energy band gap of the studied samples is also evaluated using the derivation of ineffective thickness method (DITM) (Ghobadi 2016) using Eq. (13) (Fig. 9). Figure 10 depicts the variation of $\operatorname{dln}[(A(\lambda) E)] /$ $\mathrm{d} E$ with $E$ for pristine and $\gamma$-irradiated Makrofol DE 1-1 samples. Values of the optical energy band gaps are determined and summarized in Table 1 . Results show that there is a good agreement between energy gap values

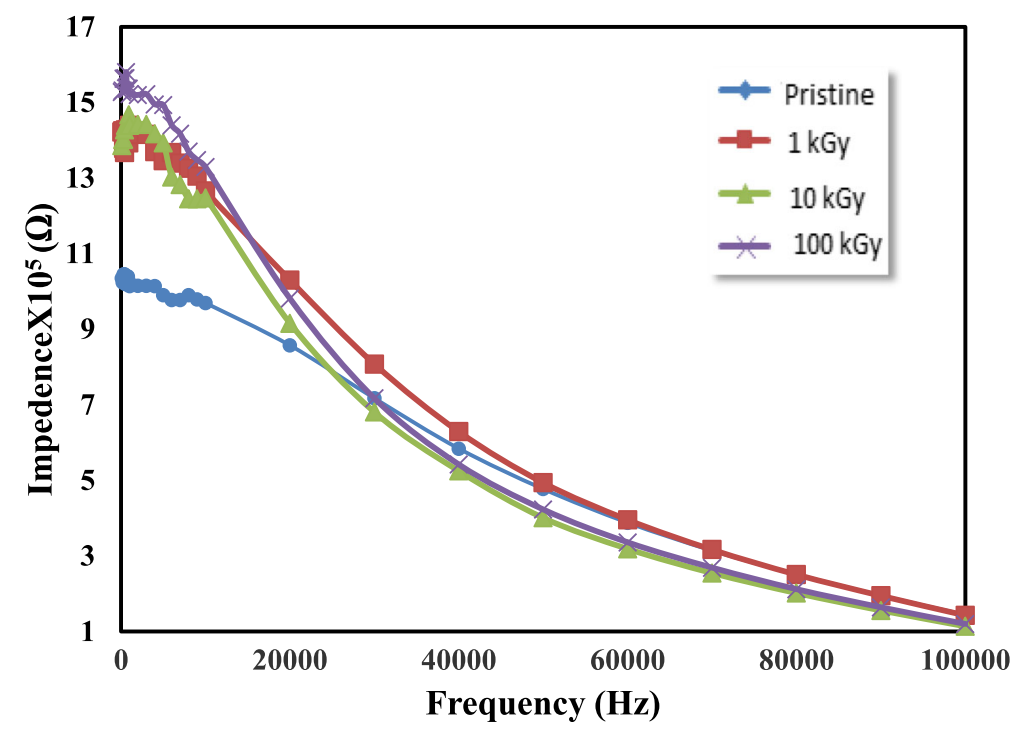

Fig. 4 Impedance versus frequency for Makrofol DE 1-1 polymer irradiated by different gamma doses 


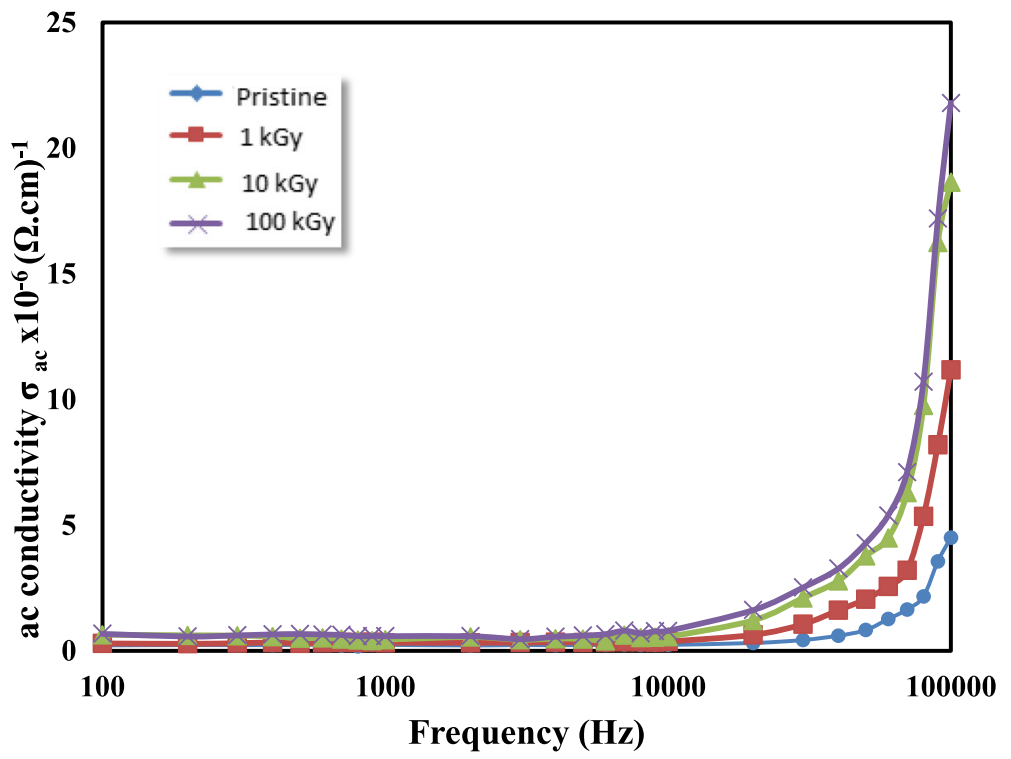

Fig. 5 Frequency dependence of the ac conductivity for the investigated samples at room temperature

which are obtained from ITM and DITM methods. One can notice that the values of energy gap of indirect and direct band gap in Makrofol DE 1-1 polymer detector decreasing with increasing gamma dose. Furthermore, the values of indirect band gap have been found to be lower than the corresponding values for the direct band gap. These decreases are attributed to the fact that irradiation induced defects and/or increase the number of conjugated bonds $(-\mathrm{C}=\mathrm{C}-)$. This means that the conductivity of the irradiated polymer with gamma radiation is greater than that of the bulk. Moreover, such a decrease in $E_{\text {optical }}$ may be attributed to the formation of new photo-chemicals, which form the trap levels between the HOMO and LUMO energy states, making the lower energy transitions feasible and results in the reduction of the optical band gap (Durrani and Bull 1987). In this study, the energy band gap for Makrofol DE 1-1 polymer decreases from 4.20 to $3.96 \mathrm{eV}$ and from 4.35 to $4.16 \mathrm{eV}$ for indirect and direct allowed transitions in ITM method, respectively. While the band gap decreases from 4.40 to $4.20 \mathrm{eV}$ in DITM method as a result of $\gamma$-ray dose increases. Thus, the present study proves that the optical energy band gap of Makrofol DE 1-1 polymer detector is rather dependent on the $\gamma$-dose. These results are somewhat consistent with the published data for the effect of $\gamma$-dose on different types of

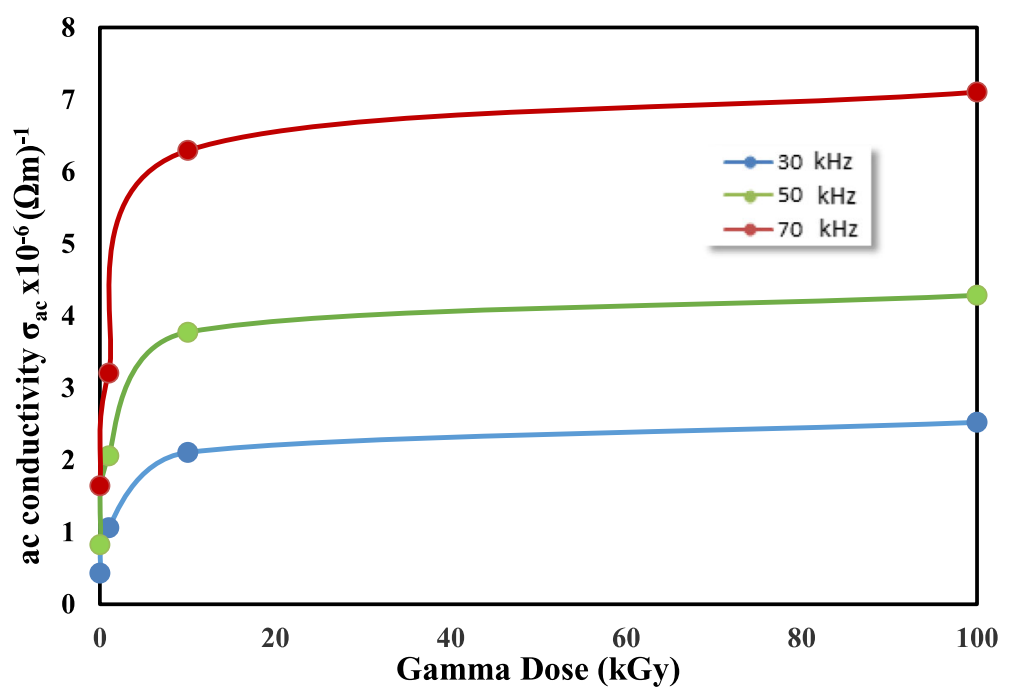

Fig. 6 The ac conductivity of the Makrofol DE 1-1 polymer versus radiation doses at different frequency values 


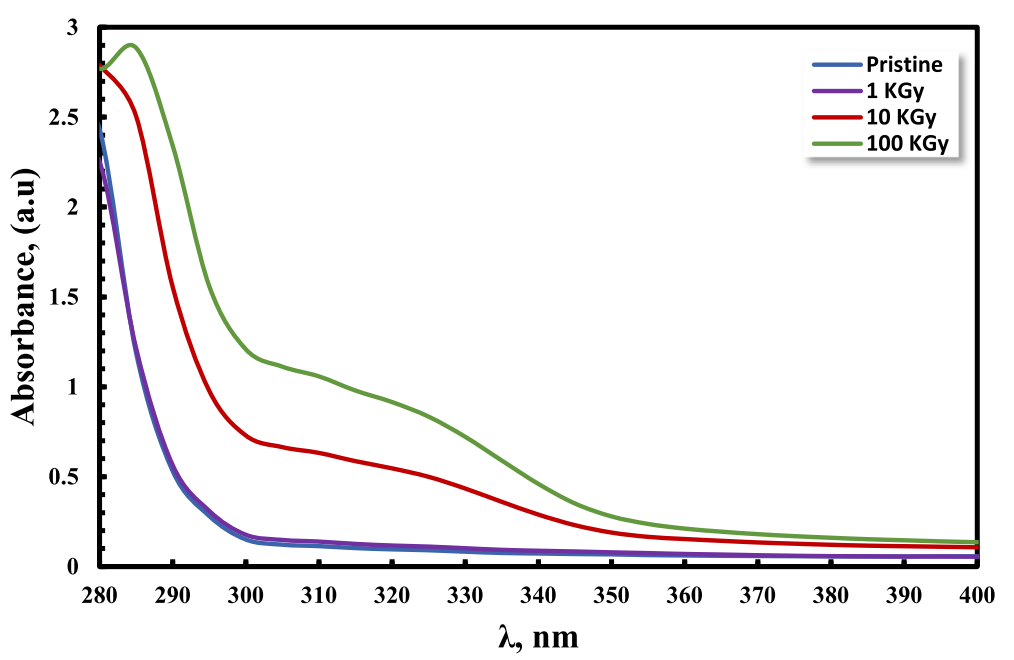

Fig. 7 UV-visible spectra of the pristine and gamma-irradiated Makrofol DE 1-1 at different doses

polymeric detectors like Sinha et al. (2001), Nouh et al. (2004), and Rammah and Abdalla (2017).

Refractive index of pristine and $\gamma$-irradiated Makrofol DE 1-1 samples was calculated using Eq. (14) (Dimitrov and Sakka 1996; ali et al. 2017). Table 1 collected the calculated refractive index by both ITM and DITM methods. Results showed that the refractive index of all samples is considerably high. The refractive index of the present samples for the direct transition is less than that for the indirect transition, and its value increases with increasing of $\gamma$-dose. This sheds light on usage $\gamma$-irradiated Makrofol
DE 1-1 detectors in optoelectronic devices, since the wave propagation is strongly influenced by the distribution of the refractive index.

Makrofol DE 1-1 is an aromatic polymer characterized by a linear chain structure, thus the optical gap band $E_{\text {opti- }}$ cal is correlated with the number of carbon bonds per molecule (Dimitrov and Sakka 1996). The shift in the absorption edges toward the visible from the ultraviolet (see Fig. 7) could be attributed to an increase in the conjugation length and a condensation of the aromatic rings into a compact carbonaceous cluster. The linear structure
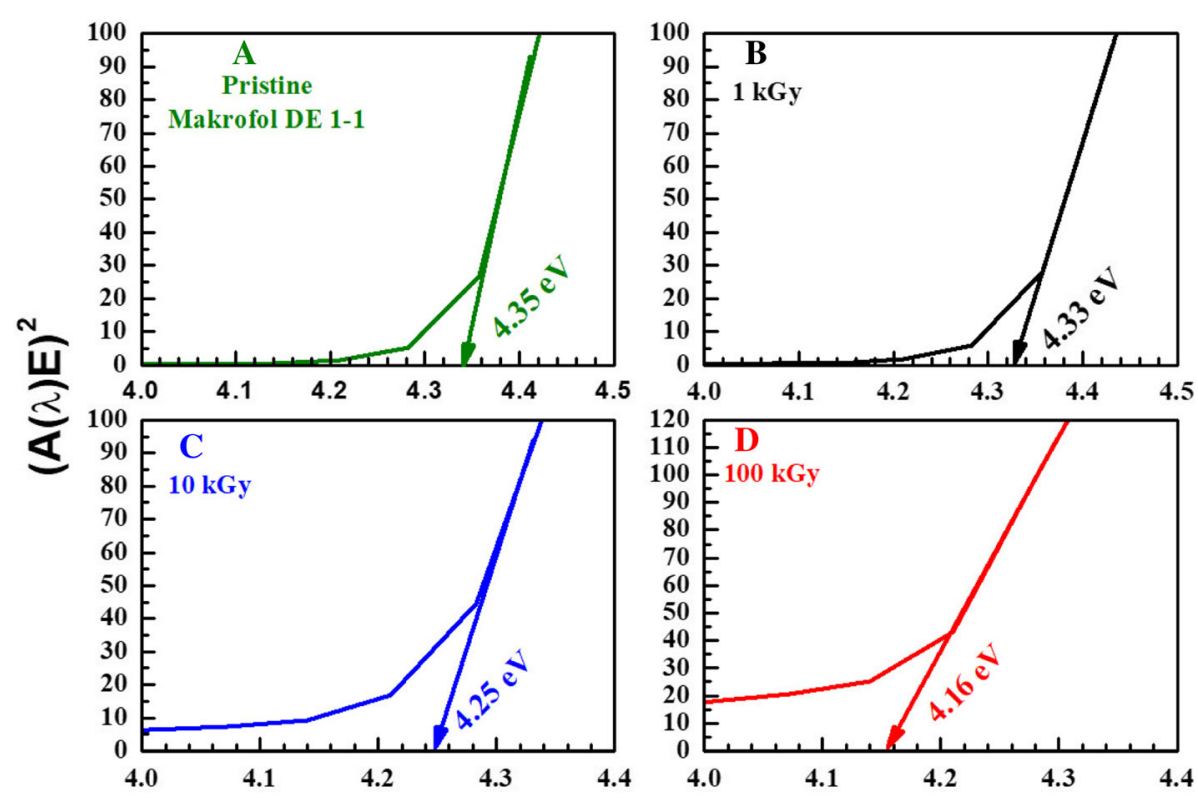

Photon energy, E (eV)

Fig. 8 Variation of $(A(\lambda) E)^{2}$ with (E): a pristine, b $1 \mathrm{kGy}, \mathbf{c} 10 \mathrm{kGy}$, and $\mathbf{d} 100 \mathrm{kGy}$ Makrofol DE 1-1 samples irradiated with gamma rays 

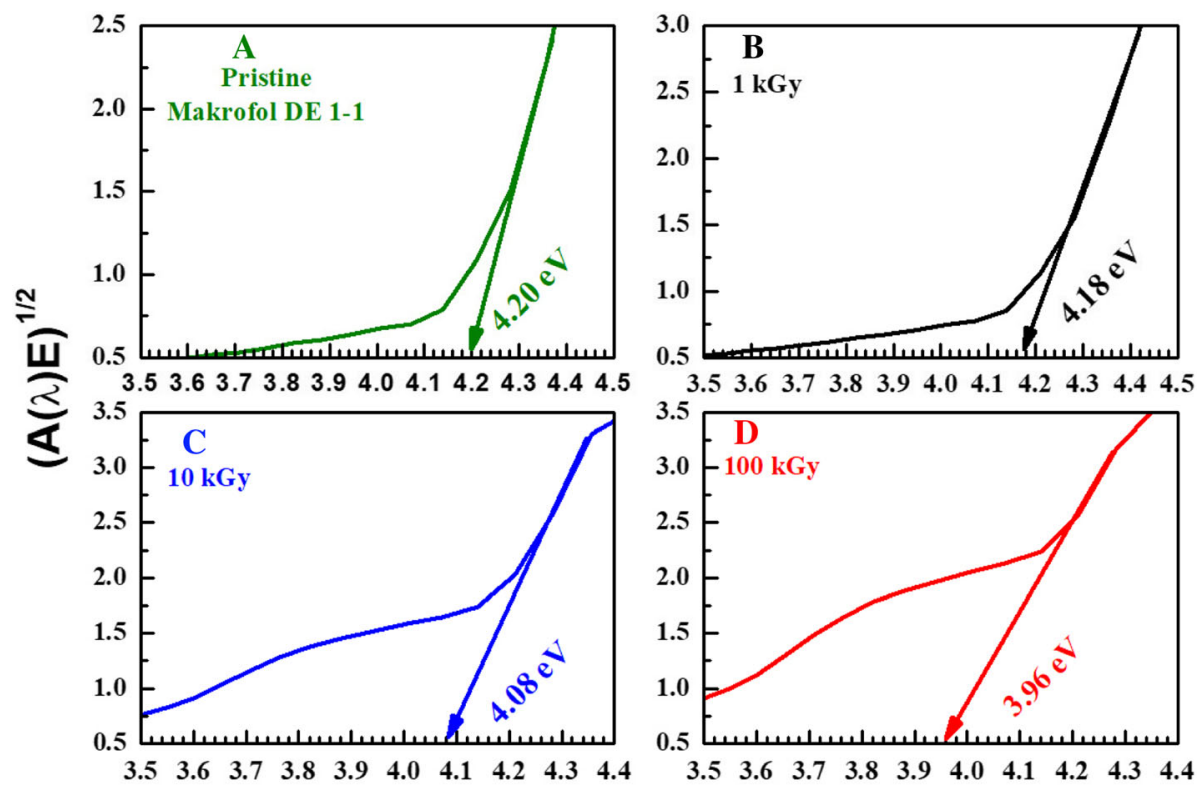

Photon energy, $\mathrm{E}(\mathrm{eV})$

Fig. 9 Variation of $(A(v) E)^{0.5}$ with $(E)$ : a pristine, b 1 kGy, c $10 \mathrm{kGy}$, and d $100 \mathrm{kGy}$ Makrofol DE 1-1 samples irradiated with gamma rays

of the polymer and the number of carbon atoms per conjugation length $N$ was calculated using Eq. (15) in direct and indirect transitions for pristine and $\gamma$-irradiated Makrofol DE 1-1 samples in ITM and DITM methods and listed in Table 2.

To enhance the optical properties of the polymeric material, the carbonaceous cluster is one of the important parameters. This cluster is rich with charge carrier, which enhances the surface conductivity of the polymers. The number of carbon atoms per cluster $M$ was calculated by Eq. (16) in direct and indirect transitions for pristine and $\gamma$-irradiated Makrofol DE 1-1 samples in ITM and DITM methods and listed in Table 2. The number of carbon atoms per conjugate length $N$ and the number of carbon atoms per cluster $M$ for the direct band gap was lower than that for the indirect energy band gap, and their values increase with increasing gamma dose. This increase is attributed to cleavages of $\mathrm{C}-\mathrm{H}$ bonds during the irradiation and, consequently, to the release of hydrogen.

\section{Conclusions}

The data analysis of the electrical parameters and the UV-visible spectra of the pristine and $\gamma$-irradiated Makrofol DE 1-1 polymeric detector led to the following conclusions:

1- The dielectric constant $\varepsilon^{\prime}$ and dielectric loss $\varepsilon^{\prime \prime}$ of pristine and irradiated samples are high at lowfrequency region nearly below $20 \mathrm{kHz}$, and their values increase with increasing $\gamma$-irradiation dose.

2- The ac conductivity of the studied polymer increases with increasing frequency and increases with increasing $\gamma$-dose.

Table 1 The ( $\left.E_{\text {optical }}\right)$ and (n) by (ITM) and (DITM) for pristine and $y$-irradiated Makrofol DE 1-1 samples

\begin{tabular}{|c|c|c|c|c|c|c|}
\hline \multirow{2}{*}{$\begin{array}{l}\text { Y- } \\
\text { irradiated } \\
\text { dose, } \mathrm{kGy}\end{array}$} & \multicolumn{2}{|c|}{$E_{\text {optical }}(\mathrm{eV}$ ) by ITM Eq. (5) } & \multirow{2}{*}{$\begin{array}{l}E_{\text {optical }} \\
\text { (eV) } \\
\text { by } \\
\text { DITM } \\
\text { Eq. } \\
\text { (13) }\end{array}$} & \multicolumn{2}{|c|}{$\begin{array}{l}\text { Refractive index, } n \\
\text { ITM }\end{array}$} & \multirow{2}{*}{$\begin{array}{l}\text { Refractive } \\
\text { index, } n \\
\text { DITM }\end{array}$} \\
\hline & Indirect & Direct & & Indirect & Direct & \\
\hline$\overline{0}$ & 4.20 & 4.35 & 4.40 & 2.13 & 2.105 & 2.09 \\
\hline 1 & 4.18 & 4.33 & 4.35 & 2.14 & 2.108 & 2.10 \\
\hline 10 & 4.08 & 4.25 & 4.30 & 2.15 & 2.123 & 2.11 \\
\hline 100 & 3.96 & 4.16 & 4.20 & 2.17 & 2.139 & 2.13 \\
\hline
\end{tabular}



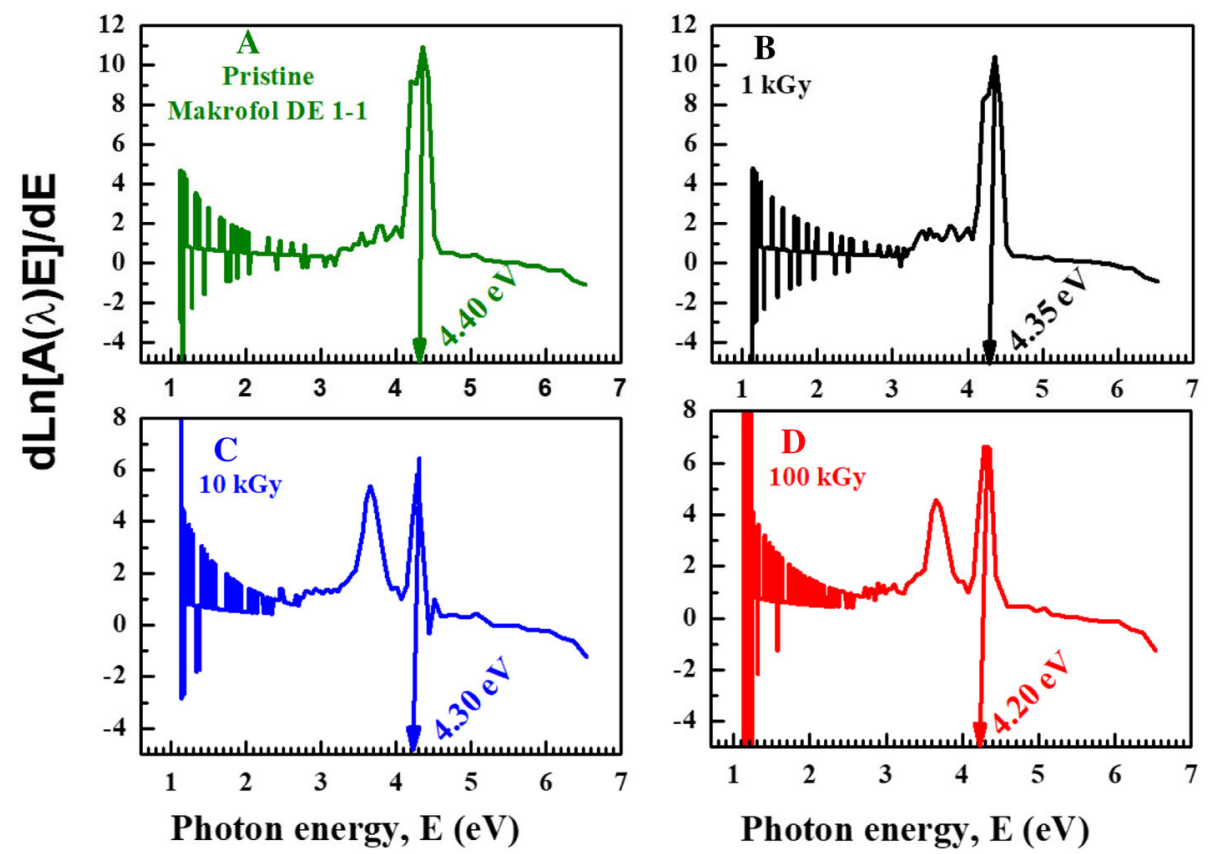

Fig. 10 Variation of $\mathrm{d} \ln [(A(\lambda) E)] / \mathrm{d} E$ with $(E)$ : a pristine, b $1 \mathrm{kGy}, \mathbf{c} 10 \mathrm{kGy}$, and $\mathbf{d} 100 \mathrm{kGy}$ Makrofol DE 1-1 samples irradiated with gamma rays

3- UV-vis spectra of pristine and $\gamma$-irradiated Makrofol DE 1-1 detector reveal a shift toward the longer wavelength with increasing of the dose.

4- Gamma-ray irradiation had a considerable effect on the optical band gap energy for Makrofol DE 1-1 detector that it decreases from 4.20 to $3.96 \mathrm{eV}$ and from 4.35 to $4.16 \mathrm{eV}$ for indirect and direct allowed transitions in ITM method, respectively. While it decreases from 4.40 to $4.20 \mathrm{eV}$ in DITM method as a result of $\gamma$-ray dose increases.

5- The refractive index of all studied samples is considerably high, its values for the direct transition is less than that for the indirect transition and increases with increasing of $\gamma$-dose.

6- The number of carbon atoms per conjugate length $N$ and the number of carbon atoms per cluster $M$ for the direct band gap are lower than that for the indirect energy band gap, and their values are increasing with increasing gamma dose.

Table 2 The $(N)$ and (M) by (ITM) and (DITM) for pristine and $\mathrm{Y}^{-}$ irradiated Makrofol DE 1-1 samples

\begin{tabular}{|c|c|c|c|c|c|c|}
\hline \multirow{2}{*}{$\begin{array}{l}\mathrm{Y}^{-} \\
\text {irradiated } \\
\text { dose, } \mathrm{kGy}\end{array}$} & \multicolumn{2}{|c|}{$\mathrm{N}$ by ITM } & \multirow{2}{*}{$\begin{array}{l}\mathrm{N} \text { by } \\
\text { DITM }\end{array}$} & \multicolumn{2}{|c|}{ M by ITM } & \multirow{2}{*}{$\begin{array}{l}\text { M by } \\
\text { DITM }\end{array}$} \\
\hline & Indirect & Direct & & Indirect & Direct & \\
\hline 0 & 4.3 & 4.18 & 4.13 & 66.69 & 62.17 & 60.76 \\
\hline 1 & 4.3 & 4.20 & 4.18 & 66.69 & 62.74 & 62.17 \\
\hline 10 & 4.4 & 4.28 & 4.23 & 70.67 & 65.13 & 63.62 \\
\hline 100 & 4.6 & 4.37 & 4.33 & 76.56 & 67.98 & 66.69 \\
\hline
\end{tabular}

7- Electrical and optical observations in this study suggested that the Makrofol DE 1-1 detector is suitable for optoelectronic and photoelectric devices.

\section{Acknowledgements}

The authors would like to thank Prof. A. Hussein, Physics Department, Faculty of Science, Menoufia University, Egypt for his fruitful help, valuable comments, and his efforts to achievement this work. Special thanks to Prof. M. El Ghazaly, Physics Department, Faculty of Science, Zagazig University, Egypt, who provides us with the samples.

Funding

No funding.

Availability of data and materials Not applicable.

Authors' contributions

All authors contributed equally in all article steps. All authors read and approved the final manuscript.

Ethics approval and consent to participate Accepted.

\section{Consent for publication}

Accepted.

\section{Competing interests}

The authors declare that they have no competing interests.

\section{Publisher's Note}

Springer Nature remains neutral with regard to jurisdictional claims in published maps and institutional affiliations. 
Received: 26 September 2018 Accepted: 11 February 2019

Published online: 20 February 2019

\section{References}

Abdul-Kader AM, Zaki MF, El-Badry BA (2014) Modified the optical and electrical properties of CR-39 by gamma ray irradiation. J Radiat Res Appl Sci 7:286-291

Abdul-Kader AM, Zaki MF, Radwan RM, Abuhadi N (2018) Influence of gamma irradiation on physical and chemical properties of Makrofol (NTD) material. Radiat Phys Chem 151:12-18

Ahmed MA, Basha AM, Marey HK, Hanafy TA (2001) Effect of neutrons and $\gamma$ radiation on cobalt-gelatin film. J Appl Poly Sci 79:1749-1755

Al-Amri A, El Ghazaly M, Abdel-Aal MS (2017) On induced-modifications in optical properties of Makrofol DE 1-1 SSNTD by UVB and UVA. Respir Physiol 7:1361-1366

Alfaramawi K, Al-Rasheedi MG, Baig MR, Alsalhi MS (2017) Solar radiation-induced changes in optical characteristics of PM-355 polymeric films. Radiat Meas 86:49-55

Ali AA, Rammah YS, El-Mallawany R, Souri D (2017) FTIR and UV spectra of pentaternary borate glasses. Measurement 105:72-77

Alsalhi MS, Baig MR, Alfaramawi K, Alrasheedi MG (2017) Influence of alpha irradiation on pre and post solar exposed PM-355 polymeric nuclear track detector sheets. Radiat Phys Chem 130:451-458

Awad EM, Soliman AA, El-Samman HM, Arafa WM, Rammah YS (2008) Alpha spectroscopy in CR-39 SSNTDs using energy simulation and matrix of energy equations for open field studies. Phys Lett A 372:2959-2966

Awad EM, Soliman AA, Rammah YS (2007) Alpha particle spectroscopy for CR-39 detector utilizing matrix of energy equations. Phys Lett A 369:359-366

Basha AM, Ahmed MA, Marey HK, Hanafy TA (1996) Neutron dosimetry measurement. Indian J Phys 70A:619-625

Chailan JF, Boiteux G, Chauchard J, Seytre G (1997) Dielectric and mechanical spectroscopies for the study of thermal and radiochemical ageing of polymers. Nucl Instr Meth B 131:172-179

Cheng S (2003) A. Annual Tech. Conference of the Soc. of Plastic Engineering (ANTEC), San Diego, Ca

Chung JYJ (1997). Stabilization of gamma-irradiated polycarbonate. Medical Plastics and Biomaterials. Technical Paper Series

Czvikovszky T, Hargitai H (1999) Compatibilization of recycled polymers through radiation treatment. Radiat Phys Chem 55:727-730

Dimitrov V, Sakka S (1996) Electronic oxide polarizability and optical basicity of simple oxides. J Appl Phys 79:1736-1740

Durrani SA, Bull RK (1987) Solid state nuclear track detection: principles and applications. Pergamon Press, Oxford

El-Sayed SM, Abdel-Hamid HM, Radwan RM (2004) Effect of electron beam irradiation on the conduction phenomena of unplasticized PVC/PVA copolymer. Radiat Phys Chem 69:339

Fares S (2011) Frequency dependence of the electrical conductivity and dielectric constants of polycarbonate (Makrofol-E) film under the effects of $y$-radiation. Amer J Mater Sci 1:52-56

Fink D, Hnatowicz V (2007) Fundamentals of ion irradiated polymers. Springer Verlag, Berlin, Germany

Ghobadi N (2016) Derivation of ineffective thickness method for investigation of the exact behavior of the optical transitions in nanostructured thin films. Mater Sci: Mater Electron 27:8951-8956

Hanafy TA (2008) Drastic effect of fast neutrons and $y$ radiation on the dcconductivity of Co-, Ni-, Mn-, and Aggelatin doped film. Curr Appl Phys 8:527-534

Hussain AMP, Saikia D, Singh F, Avasthi DK, Kumar A (2005) Effects of 160 $\mathrm{MeV} \mathrm{Ni}^{12+}$ ion irradiation on polypyrrole conducting polymer electrode materials for all polymer redox supercapacitor. Nucl Instrum Meth Phys Res B 240:834-841

Ignatius IC, Rajathi S, Kirubavathi K, Selvaraju K (2014) Studies on growth and characterization of I-alanine strontium chloride trihydrate single crystals for optical applications. Optik 125:4265-4269

Jaleh B, Nasri A, Shahbazi N, Nikfarjad H (2017) Surface properties of UV irradiated CR-39 polymer before and after chemical etching and registration of fingerprints on CR-39. Radiat Phys Chem 101:22-28

Jonscher AK (1977) The 'universal' dielectric response. Nature. 267:673-679

Kecskemeti G, Smausz T, Kresz N, Toth Z, Hopp B, Chrisey D, Berkesi O (2006) Pulsed laser deposition of polyhydroxybutrate biodegradable polymer thin films using ArF excimer laser. Appl Surf Sci 253:1185-1189
Kumar R, Virk HS, Verma KC, De U, Saha A, Prasad R (2006) Physico-chemical modifications induced in Makrofol $\mathrm{N}$-polycarbonate by swift heavy ions. Nucl Instrum Meth Phys Res B 251:163-166

Martinez-Pardoma E, Cardoso J, Vazquez H, Aquilar M (1998) Characterization of MeV proton irradiated PS and LDPE thin films. Nucl Instr Meth B 140:325-340

Mott NF, Davies EA (1979) Electronic processes in non-crystalline materials. Clarendon Press, Oxford

Nouh SA, Morsy AA, EL-Husseniy HM (2004) Radiation-induced changes in some SSNTDs. Radiat Eff Defects Solids 159:115-124

Phukan T, Kanjilal D, Goswami TD, Das HL (1999) Dielectric response of heavy ion irradiated PADC track detector. Nucl Instr Meth B 155:116-119

Price PB (2005) Science and technology with tracks in solids radiation measurements. Radiat Meas 40:146-159

Rajendran S, Sivakumar M, Subadevi R (2004) Investigations on the effect of various plasticizers in PVA-PMMA solid polymer blend electrolytes. Mater Lett 58:641-649

Rammah YS (2015) Etching and optical properties of gamma irradiated Makrofol DE 1-1 nuclear track detector. J Nucl Res Develop 10:59-62

Rammah YS (2017) Gamma radiation induced modification on optical gap of polycarbonate detectors. Amer J Opt Photonics 5:24-29

Rammah YS, Abdalla AM (2017) Study of the optical properties and the carbonaceous clusters in DAM-ADC solid state nuclear track detectors. Radiat Phys Chem 141:125-130

Rammah YS, Awad EM (2018) Modifications of the optical properties for DAM-ADC nuclear track detector exposed to alpha particles. Radiat Phys Chem 146:42-46

Rammah YS, Awad EM, Ashraf O (2018) Makrofol DE 1-4 as alpha dosimeter: optimum etching conditions. Radiat Phys Chem 151:19-24

Saad AF, Al-Faitory NM, Mohamed RA (2014) Study of the optical properties of etched alpha tracks in annealed and non-annealed CR-39 polymeric detectors. Radiat Phys Chem 97:188-197

Saad AF, Ibraheim MH, Nwara AM, Kandil SA (2018) Modifications in the optical and thermal properties of a CR-39 polymeric detector induced by high doses of $\gamma$-radiation. Radiat Phys Chem 145:122-129

Singh NL, Shah N, Singh KP, Desai CF (2005) Electrical and thermal behavior of proton irradiated polymeric blends. Radiat Meas 40:741-745

Sinha D, Phukan T, Tripathy SP, Mishra R, Dwivedi KK (2001) Optical and electrical properties of gamma irradiated PADC detector. Radiat Meas 34:109-111

Sinha D, Sahoo KL, Sinha UB, Swu T, Chemseddine A, Fink D (2004) Gamma induced modifications of polycarbonate polymer. Radiat Eff Defects Solids 159:587-595

Tauc J (1974) In: Tauc J (ed) Amorphous and liquid semiconductors. Plenum Press, New York

Tayel A, Zaki FM, El Basaty AB, Hegazy TM (2015) Modifications induced by gamma irradiation to Makrofol polymer nuclear track detector. J Adv Res 6:219-224

Velitchkova K, Krezhov K, Balabanov S (2000) Temperature dependence of surface DC electrical conductivity of carbon-implanted polymers modified by gamma irradiation. Vacuum. 58:531-535

Yoshida Y, Nishimatsu M, Mukai S, Kashiwazaki T, Yasufuku S (1980) CIGRE Poper, vol 15, p 01

Zahran AH, Ibrahim EM, Ezz-Eldin FM, El-Assy NB (1981) Electrical behaviour of $\gamma$ irradiated rigid and soft poly (vinyl chloride). Int J Appl Radiat Isot 32:713-717

\section{Submit your manuscript to a SpringerOpen ${ }^{\circ}$ journal and benefit from:}

- Convenient online submission

- Rigorous peer review

- Open access: articles freely available online

- High visibility within the field

- Retaining the copyright to your article

Submit your next manuscript at $>$ springeropen.com 